\title{
High mortality rate of obstetric critically ill women in Rwanda and its predictability
}

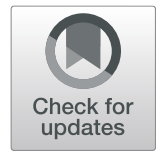

\author{
Alcade Rudakemwa $^{1^{*}}$ (D) Amyl Lucille Cassidy ${ }^{2}$ and Théogène Twagirumugabe ${ }^{3}$
}

\begin{abstract}
Background: Reasons for admission to intensive care units (ICUs) for obstetric patients vary from one setting to another. Outcomes from ICU and prediction models are not well explored in Rwanda owing to lack of appropriate scores. This study aimed to assess reasons for admission and accuracy of prediction models for mortality of obstetric patients admitted to ICUs of two public tertiary hospitals in Rwanda.

Methods: We prospectively collected data from all obstetric patients admitted to the ICUs of the two public tertiary hospitals in Rwanda from March 2017 to February 2018 to identify reasons for admission, demographic and clinical characteristics, outcome including death and its predictability by both the Modified Early Obstetric Warning Score (MEOWS) and quick Sequential Organ Failure Assessment (qSOFA). We analysed the accuracy of mortality prediction models by MEOWS or qSOFA by using logistic regression adjusting for factors associated with mortality. Area under the Receiver Operating characteristic (AUROC) curves is used to show the predicting capacity for each individual tool.

Results: Obstetric patients $(n=94)$ represented $12.8 \%$ of all 747 ICU admissions which is $1.8 \%$ of all 4.999 admitted women for pregnancy or labor. Sepsis $(n=30 ; 31.9 \%)$ and obstetric haemorrhage $(n=24 ; 25.5 \%)$ were the two commonest reasons for ICU admission. Overall ICU mortality for obstetric patients was $54.3 \%(n=51)$ with average length of stay of $6.6 \pm 7.525$ days. MEOWS score was an independent predictor of mortality (adjusted (a)OR 1.25; $95 \%$ $\mathrm{Cl} 1.07-1.46$ ) and so was qSOFA score (aOR 2.81; $95 \% \mathrm{Cl} 1.25-6.30$ ) with an adjusted AUROC of 0.773 (95\% Cl $0.67-$ 0.88 ) and 0.764 ( $95 \% \mathrm{Cl} 0.65-0.87$ ), indicating fair accuracy for ICU mortality prediction in these settings of both MEOWS and qSOFA scores.

Conclusions: Sepsis and obstetric haemorrhage were the commonest reasons for obstetric admissions to ICU in Rwanda. MEOWS and qSOFA scores could accurately predict ICU mortality of obstetric patients in resource-limited settings, but larger studies are needed before a recommendation for their use in routine practice in similar settings.
\end{abstract}

Keywords: Obstetrics, Intensive care unit, Critical care, Maternal mortality prediction

\section{Background}

Intensive care unit (ICU) admissions of obstetric patients are infrequent and little is known about incidence and outcome in low resources countries. Global incidence for obstetric patients varies from 0.4 to $16.0 \%$ of all ICU admissions [1]. In high and middle income countries, $0.13-0.76 \%$ of all births results in maternal ICU

\footnotetext{
* Correspondence: rudakal@gmail.com

${ }^{1}$ Ruhengeri Referral Hospital, North Province, Ruhengeri, Rwanda

Full list of author information is available at the end of the article
}

admission, whereas in sub-Saharan Africa (sSA) the rates are $0.24-0.95 \%$. However, only $0.65-1.5 \%$ of ICU admissions in high and middle income countries are obstetric patients compared to $1.25-6.7 \%$ in sSA [2-5].

Various reasons for admission of obstetric patients to ICUs have been identified and the prevalence of each admitting diagnosis varies between countries. Hypertensive disorders and obstetric hemorrhage are highly prevalent in high and middle income countries, whereas the most common reasons for admission in low-income countries in Africa are hemorrhage and sepsis $[2,3,5,6]$.

(c) The Author(s). 2021 Open Access This article is licensed under a Creative Commons Attribution 4.0 International License, which permits use, sharing, adaptation, distribution and reproduction in any medium or format, as long as you give appropriate credit to the original author(s) and the source, provide a link to the Creative Commons licence, and indicate if changes were made. The images or other third party material in this article are included in the article's Creative Commons licence, unless indicated otherwise in a credit line to the material. If material is not included in the article's Creative Commons licence and your intended use is not permitted by statutory regulation or exceeds the permitted use, you will need to obtain permission directly from the copyright holder. To view a copy of this licence, visit http://creativecommons.org/licenses/by/4.0/. The Creative Commons Public Domain Dedication waiver (http://creativecommons.org/publicdomain/zero/1.0/) applies to the data made available in this article, unless otherwise stated in a credit line to the data. 
Mortality among obstetric patients admitted to ICU remains obviously higher in low-income countries compared with high-income countries. While this ICU mortality was estimated at $3.5 \%$ in Netherlands, it was almost 10 times higher in Kenya and South Africa [5, 7, 8]. Predicting maternal mortality, however, remains challenging as currently available ICU severity scores are not suitable for obstetric patients admitted to ICUs such as CIPHER (Collaborative Integrated Pregnancy Highdependency Estimate of Risk) and ICNARC (Intensive Care National Audit and Research Centre). These showed high discrimination, but carry many challenges in low resource settings with limited laboratory capacities [9, 10]. Evidence showed that the Modified Early Obstetric Warning Score (MEOWS) developed by the Confidential Enquiry into Maternal and Child Health $(\mathrm{CEMACH})$ and the quick Sequential Organ Failure Assessment (qSOFA) are useful in early detection of physiological derangements [11-13]. There is no specific publication on obstetric admissions to ICU and evidence that these tools may predict outcome for obstetric patients admitted to ICU in Rwanda. Therefore, this study was conducted to determine the main reasons for ICU admission for obstetric patients, outcomes of obstetric patients admitted to ICU and to evaluate accuracy of MEOWS and qSOFA to predict mortality for obstetric patients admitted to ICU in public referral hospitals in Rwanda.

\section{Methods}

The study was conducted in the two university teaching hospitals in Rwanda: Centre Hospitalier Universitaire de Butare (CHUB) and Centre Hospitalier Universitaire de Kigali (CHUK) which are tertiary hospitals with a capacity of 454 beds, including 6 beds for ICU and 526 beds of which 7 are dedicated for ICU. We conducted a prospective cross-sectional study for all obstetric patients admitted from March 2017 to February 2018.

We included all women who were admitted to ICU with ongoing pregnancies or within 42 days after childbirth or termination of pregnancy. Patients were followed from admission to discharge from ICU. ICUtrained nurses collected data comprising demographics such as age, parity, vital signs, reason for admission recorded from ICU files, ICU interventions including inotropic agents or vasopressors needs, administration of blood components, surgical procedures, mechanical ventilation and renal replacement therapy. ICU length of stay (LOS) in days and outcome at discharge from ICU (dead or alive) were also recorded. ICU LOS was calculated as the number of days spent in ICU with zero days for a stay $>24 \mathrm{~h}$. Vital signs at admission were used to calculate MEOWS and qSOFA scores accordingly.
Data were analysed using Statistical Package for Social Sciences (IBM SPSS Statistics for Windows, version 22.0. Armonk, NY: IBM Corp). Descriptive results are reported as percentages, mean $+/$ - standard deviation and median with interquartile range (IQR). Incidence of obstetric admission to ICU was calculated based on numbers of obstetric patients admitted in ICU over numbers of all births in both hospitals during the study period. Rate of obstetric admission to ICU was based on the total number of all ICU admissions. Comparisons of frequencies or median scores between survivors and non-survivors within ICU were made by using Chisquare or Mann-Whitney $U$ tests. $P$-value $>0.05$ was considered as statistically significant. Variables with significant or a trend of association with outcome $(p<0.25)$ were included in a logistic regression model to identify independent predictors of mortality in models assessing accuracy of MEOWS or qSOFA. Generated adjusted Receiver Operating Characteristic Curves (ROC) and adjusted areas under the ROC (aAUROC) are presented. Moreover, AUROCs with $95 \%$ Confidence Intervals for each individual risk score as MEOWS or qSOFA (unadjusted analysis) are also calculated and curves provided for comparison with the adjusted ones.

\section{Results}

Demographic data and severity scores of obstetric patients on admission to ICUs

During the study period, 747 women were admitted to ICUs of either CHUB or CHUK. Of them, 94 (12.8\%) were admitted during pregnancy or within 42 days after birth or termination of pregnancy. Overall, 4,999 women were admitted for pregnancy or labor in the two facilities, corresponding to $1.8 \%$ of obstetric patients admitted to ICU.

Mean age of obstetric patients was 29.8+/-6.5 years and 52 of them $(55.3 \%)$ were admitted in their first or second pregnancy (Table 1). Seventy patients (74.5\%) were admitted after birth, 13 (13.8\%) post-abortion or following complications of ectopic pregnancies, while eleven $(11.7 \%)$ were pregnant at the time of admission to ICU. Of 70 patients admitted after birth, 44 (62.9\%) gave birth by cesarean section and 26 (37.1\%) had vaginal birth (Table 1).

\section{Reasons for admission to ICU and interventions during ICU stay}

The most common reasons for admission to ICU for obstetric patients in the two public tertiary hospitals was sepsis $(n=30 ; 31.9 \%)$, followed by obstetric hemorrhage ( $n=24 ; 25.5 \%)$, while other conditions including cardiomyopathy, stroke, embolism and trauma were diagnosed in 19 women (20.2\%). Hypertensive disorders of pregnancy $(n=$ 
Table 1 Characteristics of obstetric patients admitted in ICU in our study

\begin{tabular}{llll}
\hline Variables & Range & Frequency & \\
\hline Age (in years) & & \\
Parity at admission to ICU & $1-2$ & $52(55.3)$ \\
& $\geq 3$ & $42(44.7)$ \\
Period of admission & During pregnancy & $11(11.7)$ \\
& Post-abortion/ectopic pregnancy & $13(13.8)$ \\
& Post-partum & $70(74.5)$ \\
Admitted in post-partum period $(n=70)$ & & \\
Mode of delivery & Cesarean section & $44(62.9)$ \\
& Normal delivery & $26(37.1)$ \\
\hline
\end{tabular}

16 ; $17 \%)$ and malaria $(n=5 ; 5.3 \%)$ were the least represented (Table 2).

Mechanical ventilation was the most frequently performed intervention in $95.7 \%(n=90)$, inotropic agents and/or vasopressors were needed in $50.0 \%(n=47)$, blood components in $35.1 \%(n=33)$, surgery during ICU stay was performed on $5.3 \%(n=5)$ while hemodialysis was done in $4.3 \%(n=4)$ (Table 2). Some patients received more than one intervention as dictated by clinical conditions and/or severity of disease (Table 2).

Average length of ICU stay was $6.6 \pm 7.525$ days and $51(54.3 \%)$ study participants died in ICU.

Analysis of factors associated with ICU survival showed that neither setting nor both mode of birth and the reason for admission were associated with poorer

Table 2 Reasons for admissions and interventions performed on study patients during their ICU stay

\begin{tabular}{lll}
\hline Variables & Number of patients & Percentage (\%) \\
\hline Reason for ICU admissions & & \\
Hemorrhage & 24 & 25.5 \\
Hypertensive disorders & 16 & 17.0 \\
of pregnancy & & \\
$\quad$ Sepsis & 30 & 31.9 \\
$\quad$ Malaria & 5 & 5.3 \\
Others & $\mathbf{1 9}$ & $\mathbf{2 0 . 2}$ \\
Cardiomyopathy & 5 & 5.3 \\
Embolism & 7 & 7.5 \\
Trauma & 5 & 5.3 \\
Stroke & 2 & 2.1 \\
Interventions done & & \\
Invasive mechanical & 90 & 95.7 \\
$\quad$ ventilation & & 35.1 \\
Blood transfusion & 33 & 50.0 \\
Inotropics/vasopressors & 47 & 5.3 \\
support & & 4.3 \\
Re-operation & 5 & \\
Hemodialysis & 4 & \\
\hline
\end{tabular}

outcome (Table 3). There was, however, a statistically significant difference of MEOWS scores between survivors and non-survivors. Median MEOWS was 7 (IQR: 6; 8 ) for survivors compared with 8 (IQR: 6 ; 12) for nonsurvivors $(p=0.001)$. Also median qSOFA score was 2 (IQR: $1 ; 2)$ in the two groups. Multivariable logistic regression, adjusting for reason of admission and cesarean section before admission showed that MEOWS was an independent predictor for ICU mortality (aOR 1.25; $95 \%$ CI 1.07-1.46) and one point increase of qSOFA-score was independently associated with increased odds of ICU mortality by $181 \%$ (aOR $2.81 ; 95 \%$ CI $1.25-6.30$ ) (Table 4). In the analysis of both mortality prediction models, aAUROCs are presented in Fig. 1b. The aAUROC for MEOWS and qSOFA scores were 0.773 (95\% CI 0.666-0.880) and 0.764 (95\% CI 0.654-0.873), showing a fair discrimination capacity for mortality prediction by the two models. Besides that, unadjusted AUROCs for individual MEOWS and qSOFA scores are also presented (Fig. 1a). Unadjusted AUROC for qSOFA and for WEOWS were 0.662 (95\% CI 0.553-0.771) and 0.705 (95\% CI 0.600-0.811).

\section{Discussion}

Obstetric admissions to the ICU in public referral hospitals in Rwanda represent $12.8 \%$ of all ICU admissions and are likely to happen for $1.8 \%$ of all births in these hospitals. These rates are relatively high compared with those from high income countries, ranging between 0.22 and $0.76 \%[2,14,15]$. Our findings remain comparable to those prevailing in some middle-income countries such as Brazil (1.29\%) and Turkey (1\%) [16, 17]. Moreover, these are similar to those described in a study from Nigeria which showed that obstetric patients represented $17.3 \%$ of all ICU admissions and $2.1 \%$ of all births [18].

Rates of obstetric admissions in ICU of sSA hospitals may even be relatively higher than reported. This could be linked to limited ICU capacity as the number of ICU beds in Rwanda represents only around $1.5 \%$ of all hospital beds, whereas this number varies between 2 and 
Table 3 Factors associated with survival rate

\begin{tabular}{|c|c|c|c|c|c|}
\hline Variable & & $N=94$ & Survivors n (\%) & Non-survivors n (\%) & $p$-value \\
\hline \multirow[t]{2}{*}{ Setting } & CHUK & 60 & $25(26.6)$ & $35(37.2)$ & 0.389 \\
\hline & CHUB & 34 & $18(19.1)$ & $16(17.0)$ & \\
\hline \multirow[t]{3}{*}{ Reason for admission } & Hemorrhage & 24 & $11(11.7)$ & $13(13.8)$ & 0.078 \\
\hline & Sepsis & 30 & $9(9.6)$ & $21(22.3)$ & \\
\hline & Others & 40 & $23(24.5)$ & $17(18.1)$ & \\
\hline \multirow[t]{2}{*}{ Mode of delivery } & C-section & 44 & $24(25.5)$ & $20(21.3)$ & 0.058 \\
\hline & Vaginal & 26 & $9(9.6)$ & $17(18.1)$ & \\
\hline MEOWS [Median (IQR)] & & & $7(6 ; 8)$ & $8(6 ; 12)$ & 0.001 \\
\hline qSOFA [Median (IQR)] & & & $2(1 ; 2)$ & $2(1 ; 2)$ & 0.008 \\
\hline
\end{tabular}

$9 \%$ in majority of high income countries $[19,20]$. This challenge of scarcity of ICU beds is actually shared with other sSA countries, even if some countries may report rates between 0.24 and $0.97 \%[5,8,21]$.

The severity of conditions for obstetric patients in our study, as evidenced by the proportions of those who needed ventilator support $(90 \%)$ and inotropic agents or vasopressors $(50 \%)$ is another argument for the number of obstetric admissions in ICUs in Rwanda to be higher when capacity would allow. The two leading causes of obstetric admission to ICU in Rwanda were sepsis (31.9\%) and obstetric haemorrhagic shock (25.5\%). These conditions substantially differ in incidence from those prevailing in high income countries. Therefore, these may also partly explain discrepancies in ICU mortality given that sepsis and septic shock generally incur high mortality both in high income countries like the United States and in low income countries such as Rwanda [22, 23]. With respect of hemorrhagic shock, potential coagulation disorders resulting from delays to achieve hemostasis, lack of readily

Table 4 Multivariable Logistic regression for MEOWS and qSOFA/predictors of ICU mortality

\begin{tabular}{lll}
\hline Variables & Adjusted OR $[\mathbf{9 5} \% \mathrm{Cl}]$ & $\boldsymbol{p}$-value \\
\hline MEOWS & $1.25[1.07-1.46]$ & 0.005 \\
Caesarean & $0.39[0.12-1.22]$ & 0.106 \\
Reason for admission & & \\
$\quad$ Haemorrhage & 1 (Ref) & \\
Sepsis & $1.72[0.42-6.94]$ & 0.449 \\
$\quad$ Others & $0.65[0.18-2.39]$ & 0.517 \\
qSOFA & $2.81[1.25-6.30]$ & 0.012 \\
Caesarean & $0.33[0.11-1.02]$ & 0.054 \\
Reason for admission & & \\
$\quad$ Haemorrhage & $1($ Ref) & \\
Sepsis & $1.50[0.38-5.93]$ & 0.559 \\
Others & $0.88[0.24-3.31]$ & 0.855 \\
\hline
\end{tabular}

available blood products and massive transfusion may also explain high mortality in our setting.

ICU mortality was as high as $53.4 \%$. Relatively worse outcome has been seen in other sSA countries like Burkina Faso where this reached $60 \%$ [24]. Poor outcome of such patients may be attributable to limited management capacity of our ICUs and delays in which patients were finally admitted in severe condition. This poor outcome is shared with other ICU patients in Rwanda where mortality, among all ICU admissions, culminated in $48.7 \%$ in 2016 [23]. High rates of sepsis and associated mortality for obstetric patients in Rwandan settings have also been reported from one of the teaching hospitals [25].

Numerous tools for mortality prediction have been developed for general ICU patients such as the Acute Physiology and Chronic Health Evaluation (APACHE), the Simplified Acute Physiology Score (SAPS) and the Sequential Organ Failure Assessment (SOFA). Their generalization to obstetric patients remains challenging [26]. Our study evaluated the accuracy of easy tools such as MEOWS and qSOFA for prediction of ICU mortality in obstetric patients. The models have shown rather good discriminative capacities with fair AUROC for both models. These findings corroborate others from a study in Australia on patients suspected with sepsis, showing that a positive qSOFA $(\geq 2$ points) identified patients at higher risk of in-hospital mortality or longer ICU stay [27]. Good discriminative power of qSOFA for mortality prediction was also reported in a study from India about septic patients in ICU or non-ICU wards [28]. Findings on MEOWS are comparable with those from a study in the United Kingdom, where MEOWS had high sensitivity and good specificity to early detect morbidity among obstetric patients outside ICU [11]. Thus, MEOWS can also be applied as a simple bedside model for the purpose of predicting mortality. This, however, needs further exploration in ICU patients from various settings.

\section{Strengths and limitations}

Data for this study were prospectively collected from two tertiary hospitals that have relatively well equipped 


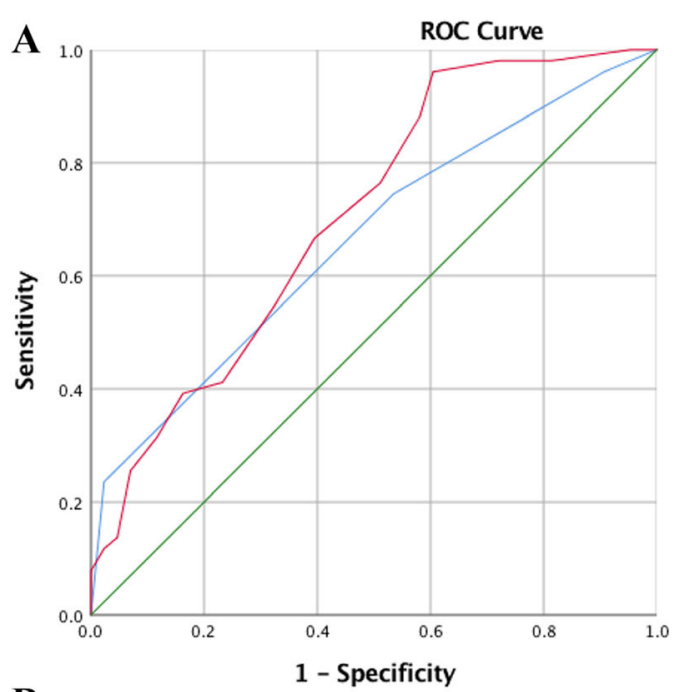

$$
\begin{aligned}
& \text { Source of the } \\
& \text { Curve } \\
& \text { - qSOFA score } \\
& \text { - MEOWS } \\
& \text { Reference Line }
\end{aligned}
$$

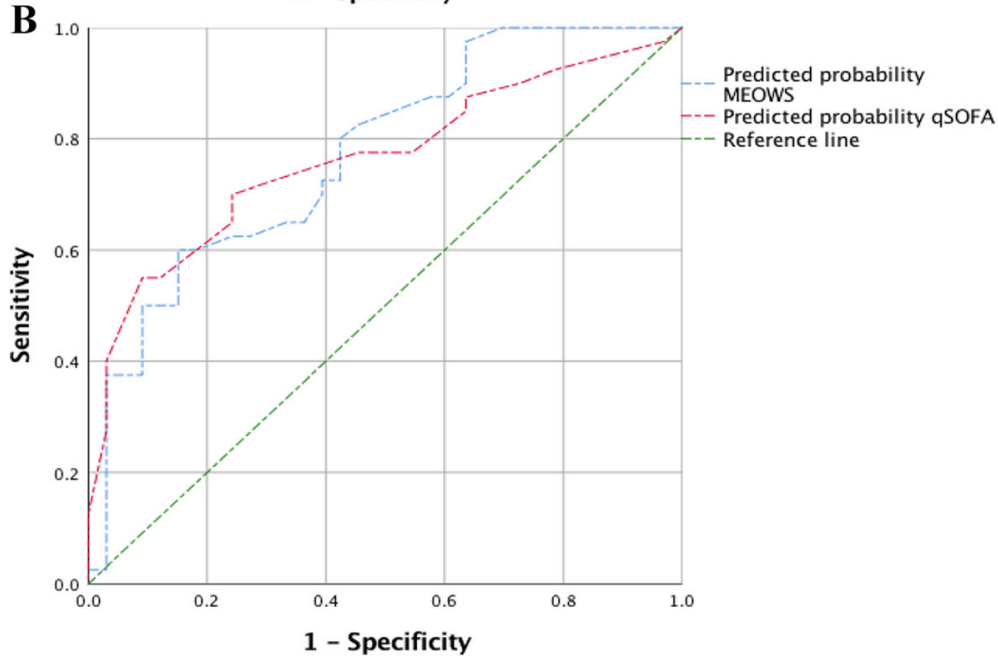

Fig. 1 a: Unadjusted AUROC for prediction of ICU mortality by qSOFA and MEOWS. b: Adjusted AUROC for prediction of ICU mortality by qSOFA and MEOWS

and staffed ICUs than other public hospitals. Therefore, majority of obstetric patients with critical conditions are admitted in those hospitals. This may give findings from the study the strength to be generalizable to obstetric ICU patients in Rwanda. The study, however, also has some limitations including small sample size. It could have been necessary to collect data for a longer period owing to the limited number of ICU beds in the country [29]. Moreover, patients were followed just during their ICU stay and outcome after discharge from ICU is another limitation. Despite that, results from this study are fundamental for further exploration to find affordable and accurate mortality prediction tools for obstetric patients admitted in ICUs of resource-limited settings.

\section{Conclusions}

Obstetric hemorrhagic shock, sepsis and septic shock are major reasons for obstetric admissions to ICUs in
Rwanda. A relatively very high ICU mortality was observed among obstetric patients. MEOWS or qSOFA scores for ICU mortality prediction in those patients perform fairly well. Further studies with larger sample size are, however, needed before generalized use of these tools for mortality prediction in critically ill obstetric patients.

\section{Abbreviations}

aOR: Adjusted Odd ratio; APACHE: Acute, Physiology, Age and Chronic Health Evaluation; AUROC: Area Under the Receiver Operating Characteristic Curve; aAUROC: Adjusted Area Under the Receiver Operating Characteristic Curve; CEMACH: Confidential Enquiry into Maternal and Child Health; CHUB: Centre Hospitalier Universitaire de Butare; CHUK: Centre Hospitalier Universitaire de Kigali; Cl: Confidence Interval; Corp.: Corporate; IBM: International Business Machines; ICU: Intensive Care Unit: IQR: Interquartile range; MEOWS: Modified Early Obstetric Warning Score; NY: New York; OR: Odd ratio; p-value: Calculated Probability; qSOFA: Quick Sequential Organ Failure Assessment; ROC: Receiver Operating Characteristic curve; SAPS: Simplified Acute Physiology Score; SCCM: Society of Critical Care Medicine; SD: Standard deviation; SOFA: Sequential Organ Failure 
Assessment; SPSS: Statistical Package for Social Sciences; sSA: Sub-Saharan Africa(n)

\section{Acknowledgements}

We are grateful to Nurses working in ICU: Mahoro Jean de Dieu of Butare University Teaching Hospital (CHUB) and Twagirimana Uzziel of Kigali University Teaching Hospital (CHUK).

\section{Authors' contributions}

AR: Study design, data analysis, interpretation and manuscript writing. $\Pi$ : Data analysis, interpretation and manuscript writing. ALC: Study design and manuscript writing. All authors have read and approved the final manuscript.

\section{Funding}

No external funding declared.

\section{Availability of data and materials}

Datasets are available from the corresponding author on reasonable request.

\section{Declarations}

\section{Ethics approval and consent to participate}

The study was reviewed and approved by the University of Rwanda/College of Medicine and Health Sciences' Institutional Review Board [approval notice No 118 /CMHS IRB/2017]. Respective hospital administrations, CHUB and CHUK, granted access to patients' data. Individual consent for participation was waived given minimal risks for patients.

\section{Consent for publication}

Not applicable. The manuscript does not contain individuals' personal data.

\section{Competing interests}

No competing interests are declared.

\section{Author details}

${ }^{1}$ Ruhengeri Referral Hospital, North Province, Ruhengeri, Rwanda. ${ }^{2}$ Department of Anesthesiology, Wake Forest University School of Medicine North Carolina, Winston-Salem, USA. ${ }^{3}$ College of Medicine and Health Sciences, University of Rwanda, University Teaching Hospital of Butare, Butare, Rwanda.

Received: 23 May 2020 Accepted: 17 May 2021

Published online: 25 May 2021

\section{References}

1. Pollock W, Dennis C. Pregnant and postpartum admissions to the intensive care unit: a systematic review. Intensive Care Med. 2010;36:1465-74.

2. Keizer JL, Zwart JJ, Meerman RH, Harinck BIJ, Feuth HDM, van Roosmalen J. Obstetric intensive care admissions: A 12-year review in a tertiary care centre. Eur J Obstet Gynecol Reprod Biol. 2006;128(1-2):152-6.

3. Leung NYW, Lau CW, Chan KKC, Yan WW. Clinical characteristics and outcomes of obstetric patients admitted to the Intensive Care Unit: a 10year retrospective review. Hong Kong Med J. 2010;16(1):18-25.

4. Bendre K, Tuteja T, Niyogi G. Critically ill obstetric patients. Int J Reprod Contraception Obstet Gynecol. 2015:4(2):370.

5. Githae F, Mung'ayi V, Stones W. Course and outcome of obstetric patients admitted to a University Hospital Intensive Care Unit. East Afr Med J. 2013; 88(10):356-60.

6. Adeniran AS, Bolaji BO, Fawole AA. Predictors of maternal mortality among critically ill obstetric patients. Malawi Med J. 2015;27:16-9.

7. Zwart JJ, Dupuis JRO, Richters A, Öry F, van Roosmalen J. Obstetric intensive care unit admission: A 2-year nationwide population-based cohort study. Intensive Care Med. 2010;36(2):256-63.

8. Ntuli TS, Ogunbanjo G, Nesengani S, Maboya E, Gibango M. Obstetric intensive care admissions at a tertiary hospital in Limpopo Province, South Africa. S Afr J Crit Care. 2015:31(1):8.

9. Payne BA, Ryan H, Bone J, et al. Development and internal validation of the multivariable CIPHER (Collaborative Integrated Pregnancy High-dependency Estimate of Risk) clinical risk prediction model. Crit Care. 2018;22(278):1-13.
10. Simpson NB, Shankar-hari M, Rowan KM, et al. Maternal Risk Modeling in Critical Care - Development of a Multivariable Risk Prediction Model for Death and Prolonged Intensive Care*. Crit Care Med. 2020;48(5):663-72.

11. Singh S, McGlennan A, England A, Simons R. A validation study of the CEMACH recommended modified early obstetric warning system (MEOWS). Anaesthesia. 2012;67(1):12-8.

12. Paternina-Caicedo A, Miranda J, Bourjeily G, et al. Performance of the Obstetric Early Warning Score in critically ill patients for the prediction of maternal death. Am J Obstet Gynecol. 2017;216(1):58.e1-58.e8.

13. Opal SM, Rubenfeld GD, Poll T, Van Der, Vincent J, Angus DC. The Third International Consensus Definitions for Sepsis and Septic Shock (Sepsis-3). JAMA. 2016;315(8):801-10.

14. Wanderer JP, Leffert LR, Mhyre JM, Kuklina EV, Callaghan WM, Bateman BT. Epidemiology of obstetric-related ICU admissions in Maryland: 1999-2008. Crit Care Med. 2013:41(8):1844-52.

15. Yi HY, Jeong SY, Kim SH, et al. Indications and characteristics of obstetric patients admitted to the intensive care unit: a 22-year review in a tertiary care center. Obstet Gynecol Sci. 2018;61(2):209-19.

16. Bandeira ARAP, Rezende CAL, Reis ZSN, Barbosa AR, Peret FJA, Cabral ACV Epidemiologic pro fi le, survival, and maternal prognosis factors among women at an obstetric intensive care unit. Int J Gynecol Obstet. 2014;124(1): 63-6.

17. Togal T, Yucel N, Gedik E, Gulhas N, Toprak HI, Ersoy MO. Obstetric admissions to the intensive care unit in a tertiary referral hospital. J Crit Care. 2010;25(4):628-33.

18. Embu HY, Isamade ES, Nuhu SI, Oyebode TA, Kahansim ML. Obstetric admissions in a general intensive care unit in north-central nigeria. Trop J Obs Gynaecol. 2016;33:14-20.

19. Murthy S, Leligdowicz A, Adhikari NKJ. Intensive care unit capacity in lowincome countries: A systematic review. PLoS One. 2015;10(1):1-12.

20. Adhikari NKJ, Fowler RA, Bhagwanjee S, Rubenfeld GD. Critical care and the global burden of critical illness in adults. Lancet. 2010;376(9749):1339-46.

21. Green K, Orazulike N. Obstetric Admission into the Intensive Care Unit (ICU) of the University of Port Harcourt Teaching Hospital: A Ten-Year Review. J Adv Med Res. 2018;25(9):1-7.

22. Hajj J, Blaine N, Salavaci J, Jacoby D. The "Centrality of Sepsis": A Review on Incidence, Mortality, and Cost of Care. Healthcare. 2018:6(3):90.

23. Riviello ED, Kiviri W, Fowler RA, et al. Predicting mortality in low-income country icus: The Rwanda mortality probability model (R-MPM). PLoS One. 2016;11(5):1-14

24. Dao B, Rouamba A, Ouédraogo D, Kambou T, Bazié AJ. Transfert de patientes en état gravido-puerpéral en réanimation: À propos de 82 cas au Burkina Faso. Gynecol Obstet Fertil. 2003;31(2):123-6.

25. Rulisa S, Umuziranenge I, Small M, van Roosmalen J. Maternal near miss and mortality in a tertiary care hospital in Rwanda. BMC Pregnancy Childbirth. 2015;15(1):1-7.

26. Id KA, Souza RD, Pinto $R$, et al. Risk prediction models for maternal mortality: A systematic review and meta-analysis. PLoS One. 2018:823:1-20.

27. Canet E, Taylor DM, Khor R, Bellomo R. qSOFA as predictor of mortality and prolonged ICU admission in Emergency Department patients with suspected infection. J Crit Care. 2018;48:118-23.

28. Maitra S, Som A, Consultant A, Bhattacharjee S, Resident S. Accuracy of quick Sequential Organ Failure Assessment (qSOFA) score and systemic inflammatory response syndrome (SIRS) criteria for predicting mortality in hospitalized patients with suspected infection: A meta-analysis of observational studies. Clin Microbiol Infect. 2018;24(11):1123-9.

29. Munyiginya P, Brysiewicz P, Mill J. Critical care nursing practice and education in Rwanda. S Afr J Crit Care. 2016;32(2):55-7.

\section{Publisher's Note}

Springer Nature remains neutral with regard to jurisdictional claims in published maps and institutional affiliations. 